\title{
The Permeation Mechanism of Cisplatin through a Dioleoylphosphocholine Bilayer
}

Lorena Ruano, ${ }^{[a]}$ Gustavo Cárdenas, ${ }^{[a]}$ and Juan J. Nogueira ${ }^{*[a],[b]}$

\author{
[a] L. Ruano, G. Cárdenas, Dr. Juan J. Nogueira \\ Chemistry Department \\ Universidad Autónoma de Madrid \\ Calle Francisco Tomás y Valiente, 7, 28049 Madrid, Spain \\ E-mail: juan.nogueira@uam.es \\ [b] Dr. Juan J. Nogueira \\ IADCHEM, Institute for Advanced Research in Chemistry \\ Universidad Autónoma de Madrid \\ Calle Francisco Tomás y Valiente, 7, 28049 Madrid, Spain
}

\begin{abstract}
The investigation of the intermolecular interactions between platinum-based anticancer drugs and lipid bilayers is of special relevance to unveil the mechanisms involved in different steps of the anticancer mode of action of these drugs. We have simulated the permeation of cisplatin through a model membrane composed of 1,2-dioleoyl-sn-glycero-3-phosphocholine lipids by means of umbrella sampling classical molecular dynamics simulations. The initial physisorption of cisplatin into the polar region of the lipid membrane is controlled, in a first moment, by long-range electrostatic interactions with the choline groups and, in a second step, by long-range electrostatic and hydrogen bond interactions with the phosphate groups. The second half of the permeation pathway, in which cisplatin diffuses through the nonpolar region of the bilayer, is characterized by the drop of the interactions with the polar heads and the rise of attractive interactions with the non-polar tails, which are dominated by van der Waals contributions.
\end{abstract}

\section{Introduction}

The biological activity of cis-diamminedichloroplatinum (II) (cisplatin) was accidentally discovered in 1965 while investigating the role of electromagnetic radiation in bacterial cell division. ${ }^{[1]}$ Subsequent tests in mice bearing sarcoma and leukemia showed remarkable tumor regression after administration of cisplatin and other platinum complexes. ${ }^{[2]}$ The first clinical tests in patients were conducted in 1971 and just seven years later cisplatin was approved by the U.S. Food and Drug Administration. ${ }^{[3]}$ Since then, an impressive amount of experimental ${ }^{[3 a, 4]}$ and theoretical ${ }^{[5]}$ work has been carried out to elucidate the mechanism of action of cisplatin, which is widely employed as chemotherapeutic drug in the treatment of patients with bladder, ovarian, head and neck, lung, testicular, cervical, esophageal, breast and brain cancers. ${ }^{[6]}$ Moreover, the success shown by cisplatin motivates the design and investigation of many other platinum-based compounds with improved biophysiological properties in the last decades. ${ }^{[7]}$

The first step of the mode of action of cisplatin and related compounds is the entry of the drug in the cancer cells. This occurs by passive diffusion through the cell membrane and by facilitated transporters, such as the CTR1 copper transport protein. $\left.{ }^{[4,}, 8\right]$ Despite its hydrophilic nature, permeability assays have shown that cisplatin is able to diffuse through the lipid bilayer due to its small size and neutral charge. ${ }^{[9]}$ Once in the cytosol, cisplatin is activated by the hydrolysis of the $\mathrm{Pt}-\mathrm{Cl}$ bonds, in which one or two chloride ligands are replaced by water to form the mono-aqua $\left[\mathrm{Pt}\left(\mathrm{NH}_{3}\right)_{2}\left(\mathrm{OH}_{2}\right) \mathrm{Cl}\right]^{+}$or the diaqua $\left[\mathrm{Pt}\left(\mathrm{NH}_{3}\right)_{2}\left(\mathrm{OH}_{2}\right)_{2}\right]^{2+}$ platinum complexes. ${ }^{[10]}$ The dissociation of the chloride ligands is favored inside the cell due to the low chloride ion concentration, which is around 13 times lower than in the extracellular fluid. ${ }^{[9]}$ After hydrolysis, the cationic platinum species are able to enter the cell nucleus and react with nucleophilic molecules, e.g., DNA strands. Specifically, the platinum atom of the hydrolyzed drug undergoes a nucleophilic attack by the N7 atoms of the purine bases, especially guanine, to form different types of DNA lesions, including monoadducts, intrastrand crosslinks and interstrand crosslinks. ${ }^{[3 \mathrm{~b}, 4 \mathrm{c}]}$ The most common DNA damage is given by the formation of $1,2-d(G p G)$ intrastrand crosslinks and, to a lesser extent, 1,2-d(ApG) intrastrand crosslinks, where cisplatin binds to two adjacent guanine nucleobases or to an adenine-guanine stacked pair, respectively. The formation of adducts between DNA and cisplatin - or other platinum complexes - induces structural distortions in the DNA helix. These helical alterations are recognized by several cellular proteins, which can initiate different complex cellular processes, including programmed cell death or apoptosis. ${ }^{[3 b, 4 c, 11]}$

One of the key steps in the mode of action of cisplatin is the transport of the drug across the membrane of the tumor cells. In fact, one possible mechanism by which cancer cells develop resistance to the chemotherapy treatment is a reduced accumulation of cisplatin, in which different cell-membrane processes are involved. $\left.{ }^{[4 c,}, 9,12\right]$ In some cancer cells, the membrane-related resistance pathways are more important than the DNA-related ones and may represent $90 \%$ of total resistance. ${ }^{[11]} \mathrm{A}$ reduced drug accumulation may be caused either by a decrease in drug uptake or by an increase in drug efflux. The passive permeation of the drug through the bilayer depends on several factors. For example, molecular dynamics simulations have evidenced that the lipid composition, the cholesterol content and the curvature of the membrane drastically affect the permeation of cisplatin. ${ }^{[5 e, 5]}$ Membrane fluidity also plays a relevant role in the passive diffusion mechanism; it has been suggested that a decrease in membrane fluidity hampers the entry of cisplatin into the cells. ${ }^{[13]}$ The fluidity of the bilayers depends, among other factors, on the insertion of molecules into the bilayer and the interactions between those molecules and the lipids. ${ }^{[4]}$ Atomic force microscopy experiments have revealed that cisplatin-encapsulated liposomes are significantly stiffer and more stable than cisplatin-free liposomes. ${ }^{[14]}$ Therefore, it could be hypothesized that the presence of cisplatin into the membrane might affect its own diffusion and uptake efficacy.

The interaction between cisplatin and membrane rafts, which can induce changes in the biophysical properties of the membrane, is not only involved in resistance mechanisms but also in different apoptotic routes. ${ }^{[4,15]}$ Although it was generally assumed that cisplatin-induced apoptosis occurs mainly through DNA adduct formation, the consideration exclusively of these DNA-mediated 
pathways is not enough to explain the toxicity exerted by cisplatin, ${ }^{33,34}$ and other mechanisms have to be invoked, including those where the lipid membrane plays a central role. Drug-lipid interactions are also relevant in the liposomal formulations of the drugs aimed to enhance the control over the delivery process and to reduce side effects and resistance of the anticancer compounds. ${ }^{[16]}$ The encapsulation of drugs inside vesicular lipid bilayers facilitates the transport of both hydrophilic and hydrophobic species, which are integrated in the polar and in the lipophilic region of the vesicle, respectively. The liposomal formulation of cisplatin, called lipoplatin, has been successfully developed and administered in clinical trials showing lower side effects than cisplatin. ${ }^{[17]}$ One possible mechanism for the delivery of the encapsulated cisplatin is the fusion of the membrane of the transport vesicle with the membrane of the cancer cell followed by the release of the drug. The efficiency of this process is largely regulated by the interactions between cisplatin and the lipids of both the carrier and the target cell.

Since cisplatin/lipid interactions play a decisive role in the initiation of apoptotic routes, resistance mechanisms and delivery of the drug from liposomal carriers, the rigorous characterization of these interactions is a necessary step towards a comprehensive understanding of the role played by lipid membranes in the mode of action of cisplatin and related species. In the present study, we unveil the intermolecular interactions that regulate the integration of cisplatin inside a model membrane composed of 1,2-dioleoylsn-glycero-3-phosphocholine (DOPC) lipids by means of umbrella sampling classical molecular dynamics simulations. Although it has been recently stated that a single-component DOPC membrane does not reproduce with accuracy the features of cisplatin permeation into more realistic cell membranes, ${ }^{[5 \mathrm{e}]}$ a DOPC membrane was chosen as a model because phosphatidylcholine is one of the major phospholipid components of the plasma membrane and of the membrane of endoplasmic reticulum, Golgi apparatus, mitochondria, endosomes and lysosomes. ${ }^{[18]}$ Due to the biological relevance of phosphatidylcholine lipids, DOPC membranes are widely employed as model in experimental and computational investigations. ${ }^{[4 \mathrm{e}]}$ However, despite the use of DOPC in a large number of publications, including theoretical modelling of the diffusion of cisplatin through the membrane, ${ }^{[16,19]}$ a detailed analysis of cisplatin/membrane interactions has never been performed even for such a simplified membrane model. Therefore, the investigation presented here is an inescapable step before tackling an analysis of the interactions between cisplatin and more complex bilayers. In addition, the use of simplified models facilitates the evaluation of the theoretical methods employed in the simulations, e.g., the accuracy of the force field.
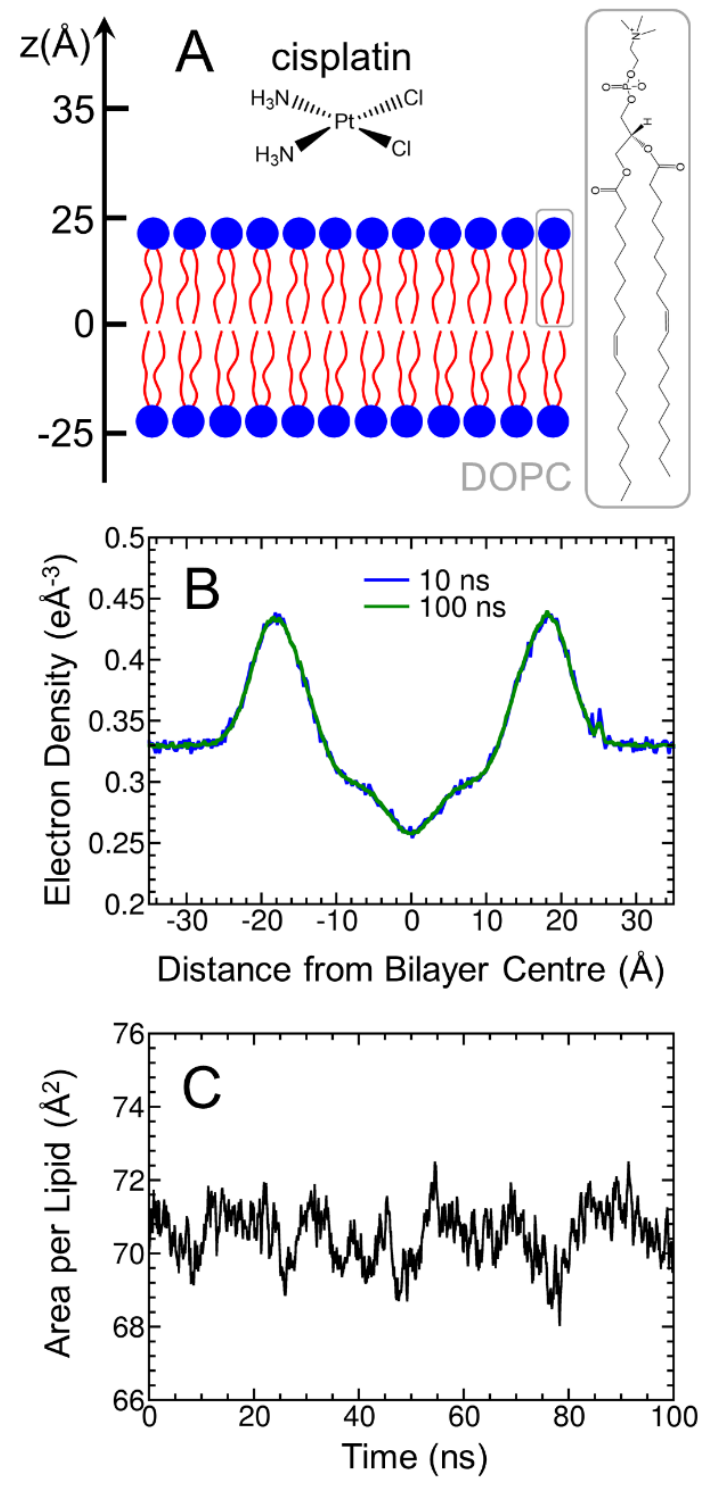

Figure 1. Analysis of the equilibration process of the DOPC membrane. (A) Schematic representation of cisplatin and the DOPC membrane. Several reference values along the $z$ axis are shown. (B) Electron density profile through the membrane computed for the first $10 \mathrm{~ns}$ (blue) and for $100 \mathrm{~ns}$ (green) during the equilibration molecular dynamics simulation. (C) Time evolution of the area per lipid along the equilibration.

\section{Results and Discussion}

\section{Equilibration and Umbrella Sampling Setup}

The diffusion of cisplatin through a lipid DOPC bilayer, schematically represented in Figure $1 \mathrm{~A}$, has been simulated by umbrella sampling molecular dynamics simulations. This enhanced-sampling approach is widely employed for modelling the slow diffusion process of small molecules across membranes ${ }^{[5 e, 5 f, 20]}$ because it allows an efficient sampling along the permeation process, which is usually very hard to simulate by conventional dynamics simulations. As first step, a conventional molecular dynamics simulation has been run to equilibrate the structure and the density of the solvated membrane and to evaluate its stability. The electron density through the lipid bilayer 
was the first property to be computed to characterize the membrane structure, and it is plotted in Figure 1B. The two peaks of the profile that appear at around -18 and $18 \AA$ in the $z$ axis indicate the position of the polar head groups of the lipid chains, which present heteroatoms $(\mathrm{O}, \mathrm{N}$ and $\mathrm{P})$ with a relatively large number of electrons, while the valley at $0 \AA$ corresponds to the centre of the bilayer, where the electron density is lower. As can be seen in Figure 1B, the electron densities computed for the first $10 \mathrm{~ns}$ of the simulation and for the entire simulation time (100 ns) are virtually the same. This indicates that the lipid molecules of the bilayer do not suffer important diffusion processes along the simulation.
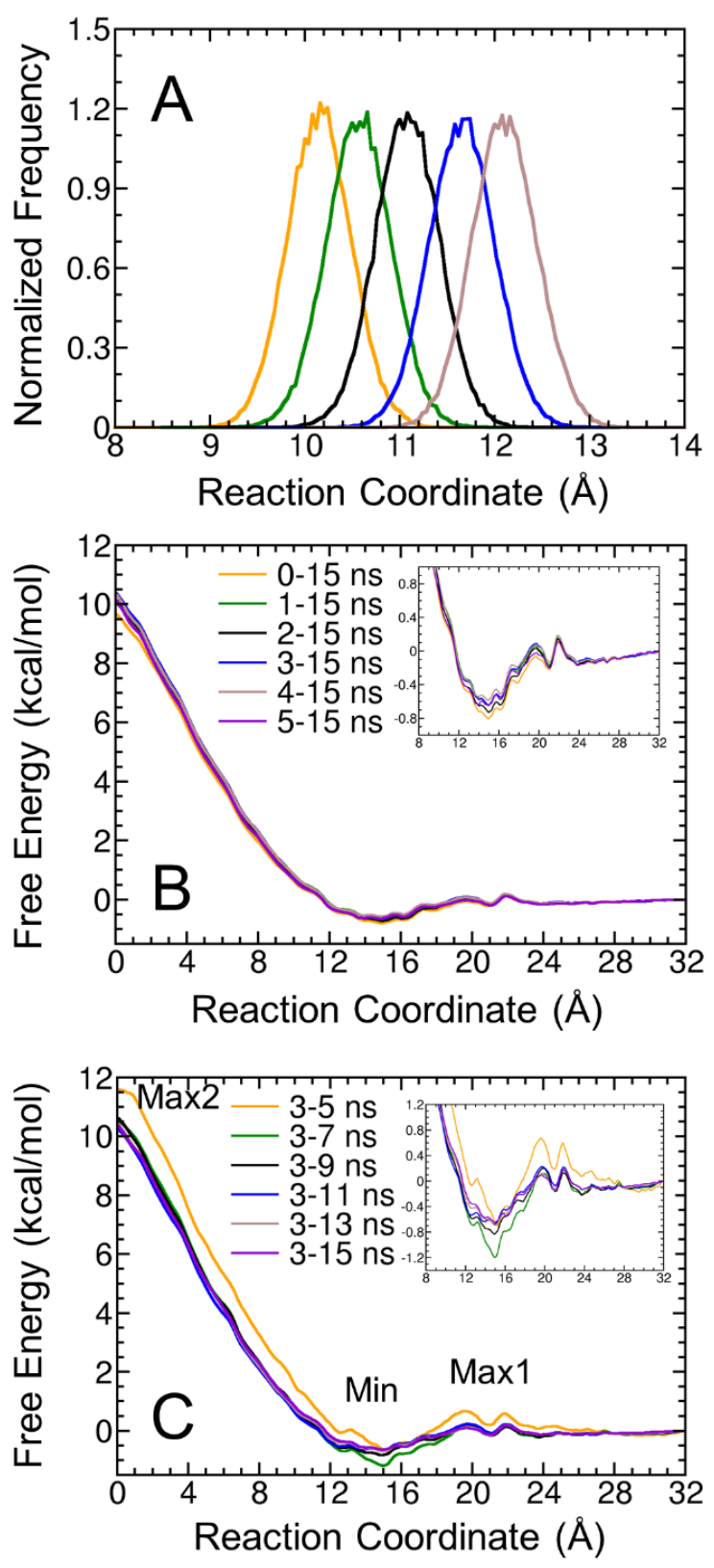

Figure 2. Convergence analysis of the umbrella sampling simulations. (A) Reaction coordinate probability distributions for the windows centred at 10.0 , $10.5,11.0,11.5$ and $12.0 \AA$. (B) Potential of mean force computed by removing different time intervals from the beginning of each window. (C) Potential of mean force computed for different computational times from the initial time of $3 \mathrm{~ns}$.
The area per lipid, which is defined as the average area that a single lipid molecule occupies on the interface, is the second property analysed to evaluate the stability of the system. Figure $1 \mathrm{C}$ shows that the area per lipid oscillates around a constant value of $70.5 \AA^{2}$ along the $100 \mathrm{~ns}$ simulation, with oscillations between 69 and $72 \AA^{2}$ and a standard deviation of $0.73 \AA^{2}$. This small oscillation amplitude also corroborates the high stability of the solvated membrane and the robustness of the theoretical model employed. In addition, the small variation underwent by both the electron density and the area per lipid with time indicates that the initial structure was already close to be equilibrated. Therefore, the solvated structure after the 100 ns molecular dynamics simulation is a good initial structure for the subsequent umbrella sampling simulations.

The reaction coordinate was defined as the separation between the centre of mass of cisplatin and the centre of mass of the DOPC membrane along the $z$ axis (see Figure $1 A$ ). The initial value of the reaction coordinate was set to $32.0 \AA$, which corresponds to a separation of around $10 \AA$ between cisplatin and the top of the surface of the bilayer. The reaction coordinate was divided into 65 windows separated by $0.5 \AA$ such that the drug diffused from the bulk solvent across half of the membrane and reached the centre of the bilayer in the last window. A value of 2.5 $\mathrm{kcal} /\left(\mathrm{mol}^{2}\right)$ was chosen as force constant for the bias potential to keep the system inside each window while allowing overlap with the neighbouring windows. It is well known that the number of windows and the force constant of the bias harmonic potential must be properly chosen to have a good overlap among the reaction-coordinate probability distributions of consecutive windows. Such a good overlap is required to obtain an accurate free-energy profile along the reaction coordinate, also called potential of mean force, from the biased probability distributions, especially when the Weighted Histogram Analysis Method (WHAM) approach is employed. ${ }^{[21]}$ Figure $2 \mathrm{~A}$ shows the computed probability distributions for the umbrella sampling windows centred at 10.0, 10.5, 11.0,11.5 and $12.0 \AA$ as example windows. It can be seen that the distributions of neighbouring windows present a strong overlap, which indicates a good sampling along the reaction coordinate and validates the choice of the simulation parameters employed.

Two additional important factors that determine the accuracy of the calculated free energy are the number of snapshots considered per window and the way they are chosen. When the reaction coordinate is modified to drive the system from one window to the next one, it is usually necessary a short equilibration process in the new window such that the system accommodates to the new value of the reaction coordinate. It is advisable to compute the free-energy profile without considering those snapshots that belong to the equilibration stage. Moreover, an accurate free-energy profile is obtained only if the simulation in each window has been run for sufficient time. These two important issues are discussed in the following analysis. Figure $2 \mathrm{~B}$ displays the potential of mean force computed for the whole simulation time ( $15 \mathrm{~ns}$ per window) and for different time intervals where the initial steps of each window were not considered; specifically, the initial 1, 2, 3, 4 and 5 ns were removed from the analysis. Note that in the profile plotted in Figure $2 \mathrm{~B}$ the reactants (cisplatin in the bulk solvent) and the products (cisplatin integrated inside the bilayer) are located on the right and on the left side of the plot, respectively. Therefore, the uptake of cisplatin in the membrane occurs from right to left. As can be seen in the inset of 
Figure $2 \mathrm{~B}$, it is necessary to eliminate the first $3 \mathrm{~ns}$ of simulation to obtain a converged profile for the relevant free-energy region located around 12-24 $\AA$, which contains the very small energy barrier (Max1) at the entrance of the bilayer and the minimum (Min). In this case, the system adapts to the new reaction coordinate value when it migrates from one window to the next one only after 3 ns are elapsed. Therefore, in the subsequent analyses the first 3 ns of each window will not be considered. Next, we analyse for how long each window must be evolved to get a converged free-energy profile. Figure $2 \mathrm{C}$ shows that convergence is achieved after running $8 \mathrm{~ns}$ (from $3 \mathrm{~ns}$ to $11 \mathrm{~ns}$ ) of dynamics simulation per window. Therefore, it is not necessary to extend the simulations for longer time than the $15 \mathrm{~ns}$ per window initially run. The energetic analysis discussed below will be performed by taking into account snapshots from the time interval from 3 to 15 ns in each window.
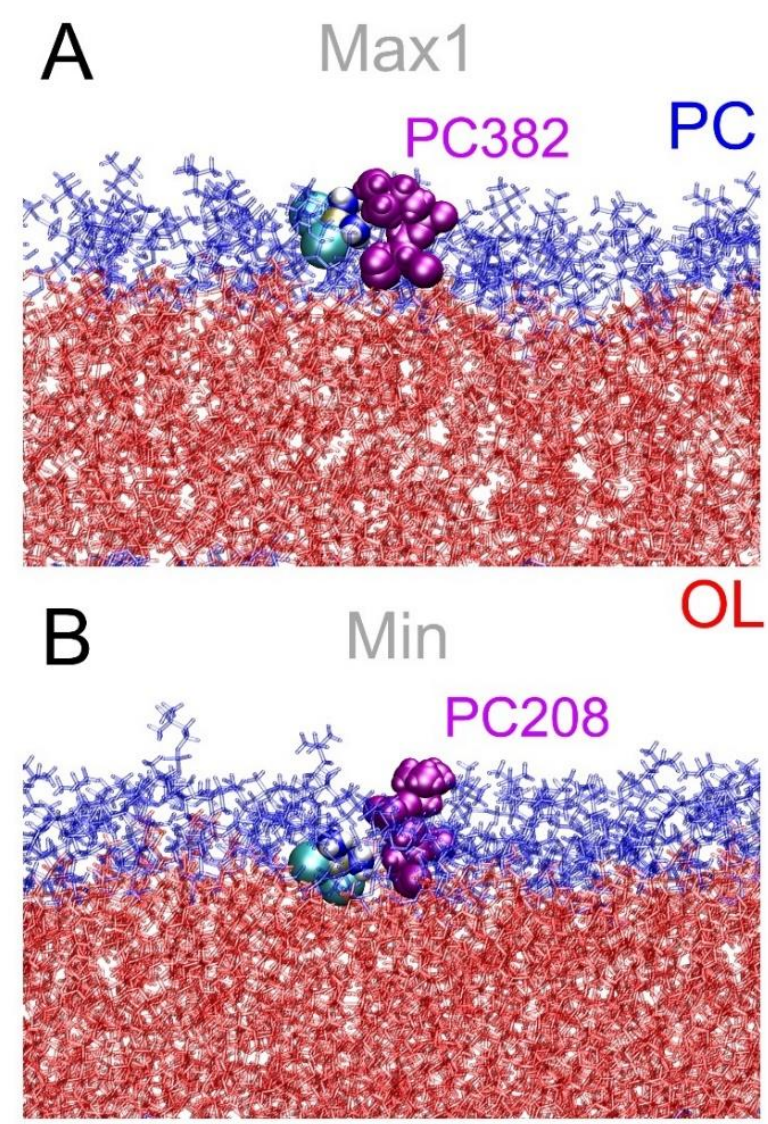

Figure 3. Representative snapshot of cisplatin interacting with the residue PC382 at Max1 (A) and with the residue PC208 at Min (B). Cisplatin is represented with van der Waals spheres with the following atom colour code: ochre for $\mathrm{Pt}$, blue for $\mathrm{N}$, white for $\mathrm{H}$ and cyan for $\mathrm{Cl}$. The residues $\mathrm{PC} 382$ and PC208 are represented by magenta van der Waals spheres. The polar heads $(\mathrm{PC})$ and the nonpolar tails $(\mathrm{OL})$ of the membrane are represented by blue and red sticks, respectively.

\section{Characterization of Intermolecular Interactions}

The potential of mean force presents one energy minimum and two energy maxima - called Min, Max1 and Max2 in Figure 2C that will be analysed in detail in the following. When cisplatin approaches the membrane, there is an initial attraction between the drug and the bilayer which results in a free-energy minimum (Min) at $15 \AA$ of $0.65 \mathrm{kcal} / \mathrm{mol}$ depth with respect to the energy of cisplatin located in the bulk solvent. This free-energy minimum corresponds to the physisorption of cisplatin on the polar region of the membrane. The minimum is reached after overcoming a very small free-energy barrier (Max1) of around $0.2 \mathrm{kcal} / \mathrm{mol}$ extended along the free-energy curve region located between reaction coordinate values of 19 and $23 \AA$. However, it is not clear whether this slight energy increase corresponds to an actual energy barrier or whether it is noise, whose fluctuations lie within the error of the method. In any case, the available thermal energy is enough to drive the system through this region of the freeenergy surface and accommodate the drug inside the free-energy minimum. If cisplatin continues the diffusion pathway towards the center of the bilayer the free energy steadily increases until achieving its absolute maximum (Max2) in the middle of the membrane, which is $10.4 \mathrm{kcal} / \mathrm{mol}$ higher than the energy of the global minimum Min2. This energy barrier agrees very well with previous dynamic simulations, where an energy barrier of around $10 \mathrm{kcal} / \mathrm{mol}$ was obtained. ${ }^{[5 e, 19]}$ Moreover, our simulations are also in good agreement with experimental findings. Specifically, the permeability coefficient of cisplatin travelling through DOPC vesicles obtained by kinetic measurements was $1.1 \times 10^{-8} \mathrm{~ms}^{-1}$ at a $\mathrm{Cl}^{-}$concentration of $0.15 \mathrm{M},{ }^{[9]}$ while the permeability coefficient computed from the autocorrelation function of the Z-position of the permeating drug, as previously explained, ${ }^{[20 c]}$ extracted from our simulations is $3.6 \times 10^{-7} \mathrm{~ms}^{-1}$. Once the results from the simulations have been validated with previous calculations and experiments, the behavior of cisplatin inside the DOPC bilayer and the intermolecular interactions that lead to the permeation of the drugs will be analyzed below.

A visual inspection of the umbrella sampling windows located at Max1 and Min reveals that the environment and the behavior of cisplatin is different in both regions. While cisplatin is located at the water/membrane interface at Max1 (Figure $3 A$ ), it is completely inside the polar region of the bilayer formed by the phosphocholine (PC) groups at Min (Figure 3B). This situation results in a higher mobility and a shorter interaction time with the lipids when the drug is located at the more external region Max1 than when it is at Min. To illustrate this, we show in Figure 4 the time evolution of the number of interatomic contacts between cisplatin and the five lipid residues that present the highest interaction energy with the drug in the umbrella windows located at 20 and $15 \AA$, which correspond to the free energy regions Max 1 and Min, respectively. These residues are the PC polar heads 358 , 382, 307, 385 and 373 for Max1, and the PC residues 343, 208, 328 and 196 and the nonpolar dioleoyl (OL) tail 207 for Min. A contact between a cisplatin atom and a lipid atom is arbitrarily considered when the separation between both atoms is lower than $5 \AA$. As can be seen, interatomic contacts between cisplatin and lipid residues are present for shorter time at Max1 than at Min. For example, if one considers that the contact between the drug and $\mathrm{PC}$ or $\mathrm{OL}$ residues is present when the number of interatomic contacts is 10 or higher, the lifetime of the intermolecular contacts is between 6 and $10 \mathrm{~ns}$ for Min, but only between 4 and $6 \mathrm{~ns}$ for Max. This indicates that the motion of the drug is restrained when it is located at the free-energy minimum Min, while it presents a higher degree of freedom when it is on the region Max1. 


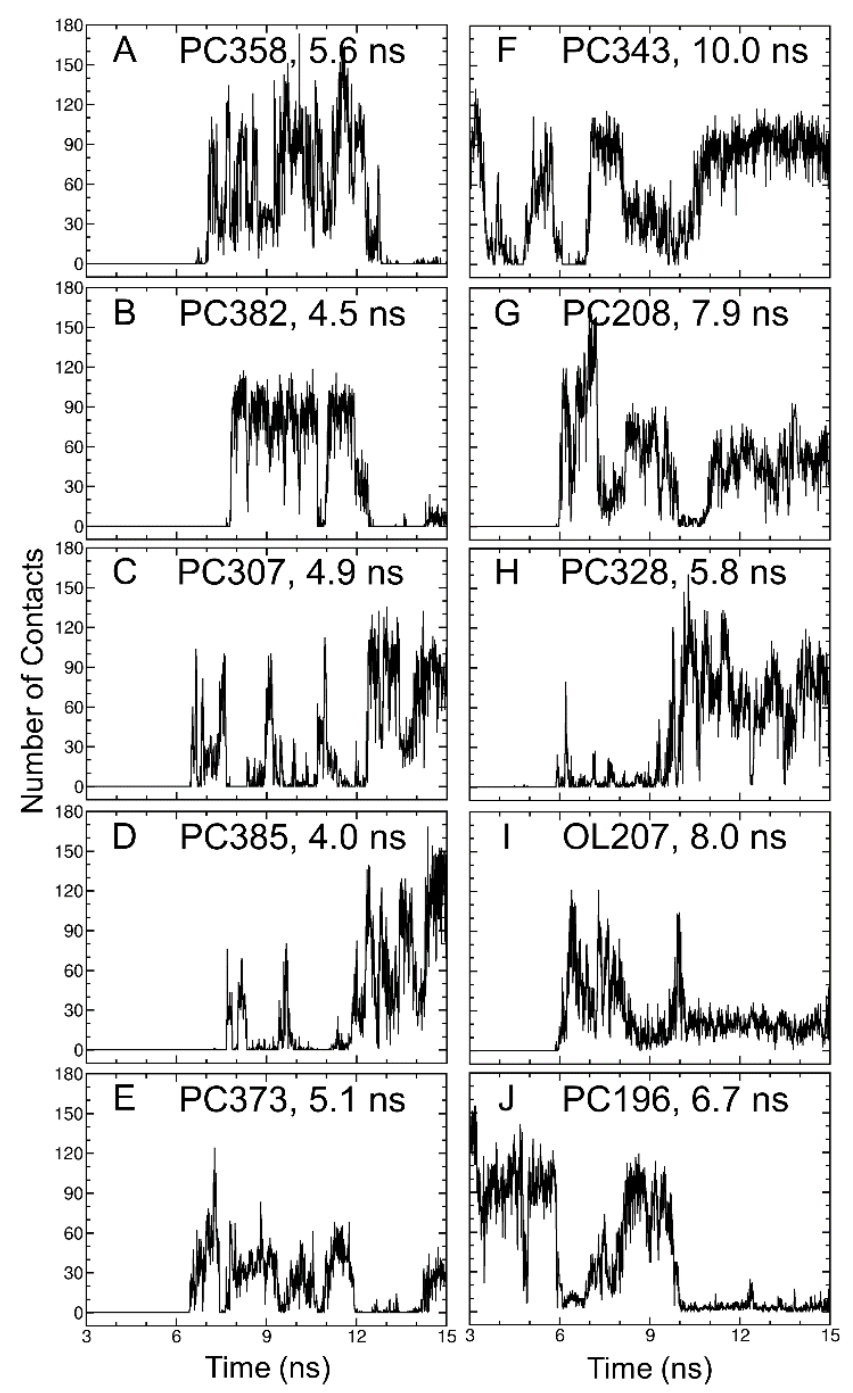

Figure 4. Time evolution of the number of interatomic contacts between cisplatin and the five residues that present the highest interaction energy with the drug at Min (A-E) and Max1 (F-J), at the umbrella-sampling windows located at 15 and $20 \AA$, respectively. The lifetime of the interaction with each of the residues is also shown. It is assumed that the interaction is present when the number of interatomic contacts is 10 or higher.

To get more insight on the nature of the intermolecular interactions that drive the diffusion of cisplatin through the lipid bilayer the interaction potential energy between cisplatin and the membrane was decomposed into different contributions along the permeation pathway. First, we analyze the contribution of the polar PC heads and nonpolar OL tails to the total interaction energy. The different chemical groups that belong to these two regions of the lipid chains are shown in Figure 5A. In addition, each of these contributions (PC heads and OL tails) can be further decomposed into van der Waals and electrostatic interactions, which in the force field employed here are described by LennardJones and Coulomb potentials, respectively. As seen in Figure $5 B, C$ the interaction energy between cisplatin and the PC head groups dominates along the entire reaction coordinate over the interaction between cisplatin and the $\mathrm{OL}$ tails, except in the region of the absolute energy maximum (Max2), where the $\mathrm{OL}$ contribution is more important than the PC one.
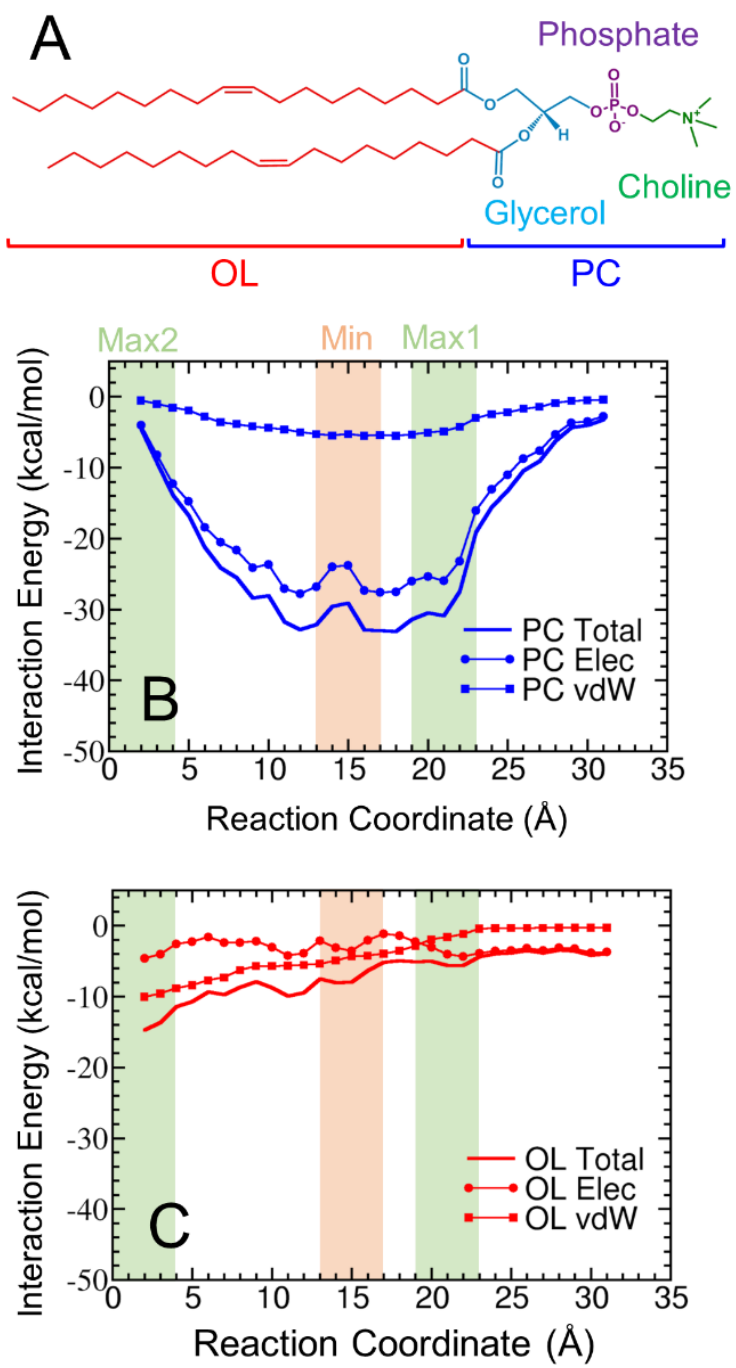

Figure 5. Contribution of phosphocholine (PC) head groups and dioleoyl (OL) tails to the cisplatin/membrane interaction energy along the reaction coordinate. (A) Different chemical groups of the DOPC lipid chains. (B) Interaction energy between cisplatin and the PC groups, and decomposition into electrostatic $(E l e c)$ and van der Waals (vdW) contributions. (C) Interaction energy between cisplatin and the OL groups, and decomposition into electrostatic (Elec) and van der Waals (vdW) contributions.

The cisplatin/PC interaction energy steadily increases (in absolute value) along the permeation pathway until the drug reaches the minimum (Min), where the attractive interaction between the drug and the head groups achieves a value around $-35 \mathrm{kcal} / \mathrm{mol}$. Then, along the pathway from Min to Max2, the cisplatin/PC interaction becomes less attractive with values between -20 and $-30 \mathrm{kcal} / \mathrm{mol}$ in regions around reaction coordinate values of $10 \AA$, and between -10 and $-5 \mathrm{kcal} / \mathrm{mol}$ at the middle of the bilayer (Max2). Figure $5 \mathrm{~B}$ also shows that the interaction between the drug and the polar heads is clearly dominated by electrostatic interactions, which represent $85 \%$ of the cisplatin/PC interaction energy (as average along the entire permeation pathway), while the van der Waals contribution represents only $15 \%$.

The value of the cisplatin/OL interaction attractive energy steadily increases when the drug diffuses from the bulk solvent to the middle of the membrane, where it reaches a value of around -15 
$\mathrm{kcal} / \mathrm{mol}$. Contrary to the cisplatin/PC energy, the most important contribution to the interaction between cisplatin and the nonpolar tails is given by the van der Waals interactions, especially on the region between both free-energy maxima where the van der Waals interactions constitute $73 \%$ of the total energy. Since the cisplatin/OL attraction steadily increases along the diffusion pathway, but the cisplatin/PC attraction first increases until Min, and then decreases from Min to Max2, the first half of the permeation pathway is regulated by electrostatic interactions, while in the second half of the pathway the van der Waals interactions gain relevance. Specifically, the van der Waals interaction represents only $27 \%$ of the total interaction energy at the umbrella sampling window located at $15 \AA$ (corresponding to Min), while it is $52 \%$ of the total energy at the window located at 0 $\AA$ (corresponding to Max2).

Since the PC heads are formed by different chemical groups, namely choline, phosphate, and glycerol groups (see Figure 5A), it is interesting to evaluate the contribution of each of these moieties to the cisplatin/PC interaction energy along the reaction coordinate and, especially, around the Max1-Min free-energy curve region, where the initial physisorption process occurs. Such an energy decomposition is displayed in Figure 6 . As can be seen, the glycerol groups play an almost irrelevant role along the entire reaction pathway in comparison to the choline and phosphate groups. For these last two groups, the electrostatic interactions with cisplatin largely surpass the van der Waals ones, as was already evidenced when discussing the interaction energy of the entire PC head (Figure 5B). The interaction with the phosphate units is slightly repulsive on top of the DOPC membrane (reaction coordinate between 23 and $27 \AA$ ), while the attraction with the choline groups counteracts the repulsion with the phosphate units, stabilizing the system. Then, when the drug is on the region of Max1 the attraction with the choline residues is maximum and the interaction with the phosphates is also attractive but smaller. From Max1 the cisplatin/choline attraction steadily decreases until the interaction becomes repulsive at Min, while the cisplatin/phosphate attraction steadily increases until the drug reaches the Max2 region. At the minimum (Min), the attractive interactions between cisplatin and the phosphate groups clearly dominate the system. Therefore, it can be stated that the electrostatic attractive interactions between cisplatin and choline drive the initial approach of the drug to the bilayer and then, the electrostatic attraction with the phosphate moieties is responsible for the deeper integration of cisplatin in the free-energy minimum. From Min to Max2, the attraction exerted by the phosphate groups is partially compensated by the repulsion with the choline units, while the van der Waals interactions with the nonpolar tails gain importance, as seen in Figure 5B.

Finally, the large number of heteroatoms present in the $\mathrm{PC}$ heads (see Figure $5 \mathrm{~A}$ ) could lead to the formation of hydrogen bonds with cisplatin. Specifically, the amino groups of cisplatin (see Figure $1 \mathrm{~A}$ ) could participate as hydrogen donors and the oxygen atoms of phosphate and glycerol residues as hydrogen acceptors. The positive charge of the nitrogen atom of choline precludes the participation of this atom in hydrogen bond interactions. The occurrence of hydrogen bonding with the glycerol and phosphate moieties - defined as the percentage of the simulation time that a hydrogen bond is present - was analyzed along the permeation pathway. It was assumed that a hydrogen bond is formed when the distance between the hydrogen donor and the hydrogen acceptor is shorter than $3.0 \AA$, and the angle formed by the hydrogen donor, hydrogen, and hydrogen acceptor is larger than $135^{\circ}$, which are arbitrary criteria commonly used in the literature. ${ }^{[22]}$ Figure $6 \mathrm{D}$ shows that the presence of hydrogen bonding with the phosphate groups is relevant during the initial physisorption of the drug around the Max1 and Min regions, while the occurrence of hydrogen bonding with glycerol is important at a later stage between Min and Max2. However, the weak electrostatic interactions observed in Figure 6C indicates that, although the presence of hydrogen bonds between cisplatin and glycerol moieties is relatively high, these hydrogen bonds are not strong and, therefore, do not play a relevant role in the transport of cisplatin through the membrane.
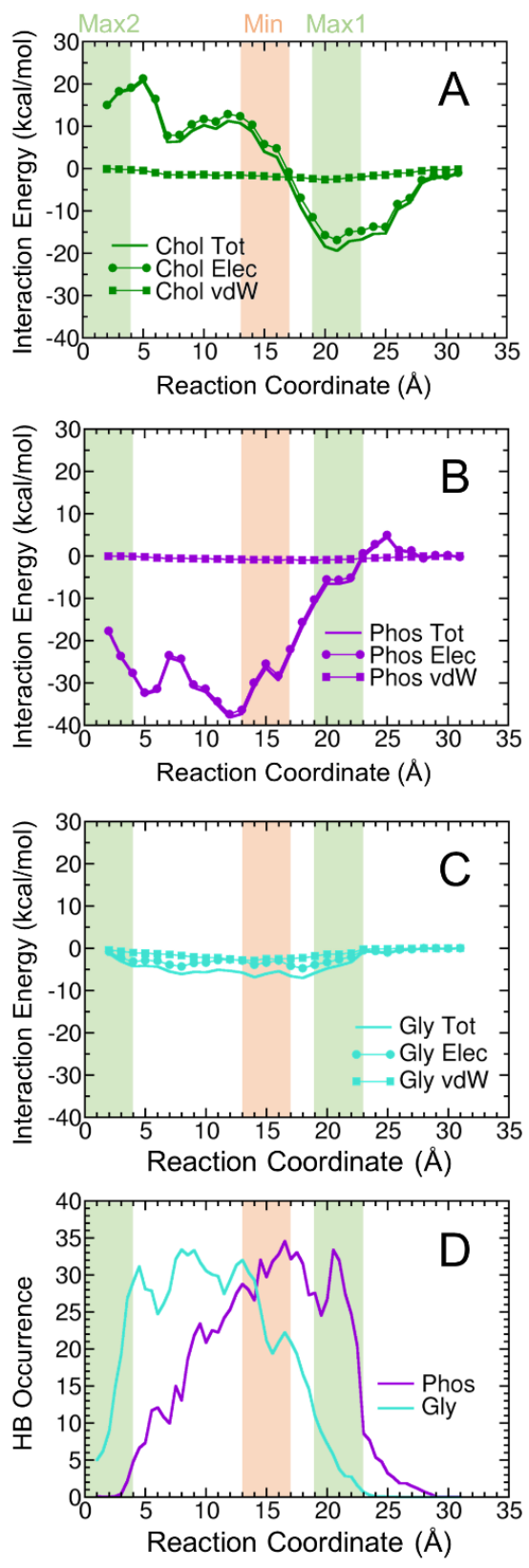

Figure 6. Decomposition of the interaction energy between cisplatin and phosphocholine (PC) groups. Interaction energy between cisplatin and the choline (A), phosphate (B), and glycerol (C) groups, together with their van der Waals and electrostatic energies decomposition, along the permeation pathway. (D) Hydrogen bond (HB) occurrence between cisplatin and the phosphate and glycerol groups along the permeation pathway. 


\section{Conclusion}

The intermolecular interactions between cisplatin (and other platinum-based drugs) and cell membranes play an important role on different steps of the mode of action of these anticancer drugs, including the uptake process, resistance mechanisms and initiation of apoptosis. In addition, these interactions are also relevant on the delivery of the drug from liposomal carriers of encapsulated formulations. Therefore, the investigation of the nature of the intermolecular interactions present in cisplatin/membrane systems is crucial to gain knowledge that can contribute towards the understanding of the molecular mechanism behind the mode of action of platinum drugs and towards the design of new compounds with improved properties. In this work, we have simulated the diffusion process of cisplatin trough a DOPC lipid bilayer by means of umbrella sampling molecular dynamics simulations to unveil the mechanism of permeation of cisplatin through the lipid membrane.

Cisplatin is first weakly trapped on a free-energy minimum of 0.65 $\mathrm{kcal} / \mathrm{mol}$ depth after overcoming a very small energy barrier of 0.2 $\mathrm{kcal} / \mathrm{mol}$. At this minimum, cisplatin is completely integrated inside the polar region of the bilayer and presents a lower mobility than when it is around the barrier region, which corresponds to the interface between the solvent and the membrane. This is reflected in the longer lifetime of the interactions between cisplatin and specific polar heads when the drug is trapped in the minimum. During this initial stage, where cisplatin is around the first maximum and the minimum of the free-energy surface (first half of the permeation pathway), the integration of cisplatin into the membrane is driven first by electrostatic interactions with the choline groups and then by electrostatic interactions with the phosphates. The formation of hydrogen bonds with the oxygen atoms of phosphate groups contributes to these electrostatic interactions since hydrogen bonds are present during $30 \%$ of the simulation time. Along this first half of the permeation pathway the interaction of cisplatin with the glycerol moieties of the polar heads and with the non-polar tails is insignificant. In addition, the more important interactions with the choline and phosphate units are largely dominated by the electrostatic component (around 85\%), while the van der Waals contribution is only minor (around 15\%). Along the second half of the permeation pathway, where the drug moves from the minimum to the global maximum located at the middle of the membrane, the cisplatin/choline repulsion and the cisplatin/phosphate attraction steadily increase and partially cancel each other out. This partial cancelation induces a reduction of the total interaction energy between cisplatin and the head groups. This is accompanied by an increase of the attractive interactions between cisplatin and the non-polar tails, which are dominated by the van der Waals contribution, contrary to the interactions with the polar heads. Therefore, van der Waals interactions gain importance in the non-polar region of the membrane and represent more than $50 \%$ of the total cisplatin/membrane interaction energy. The energy barrier that the system must overcome to reach the global maximum is 10.4 $\mathrm{kcal} / \mathrm{mol}$, a value which agrees very well with previous simulations ${ }^{[5 e, 19]}$ and experimental measurements. ${ }^{[9]}$

\section{Computational Details}

The lipid bilayer formed by 64 molecules of DOPC per layer with a $25 \AA$ of water thickness on each side was built with the help of the CHARMM-GUI website. ${ }^{[23]}$ Furthermore, a concentration of $0.15 \mathrm{M}$ of $\mathrm{KCl}$ was added to reproduce the physiological concentration of this salt. Then, cisplatin was manually placed at a distance of $32 \AA$ from the centre of mass of the membrane using the tleap module of AmberTools19, ${ }^{[24]}$ resulting in a system of 35451 atoms. The lipid bilayer was described by the Lipid17 force field, which is an update of the previously developed Lipid11 ${ }^{[25]}$ and Lipid14 ${ }^{[26]}$ force fields for lipids. Water molecules were described by the TIP3P mode ${ }^{[27]}$ and the $\mathrm{K}^{+}$and $\mathrm{Cl}^{-}$ions by suitable Amber parameters. ${ }^{[28]}$ The bond and angle parameters of cisplatin were obtained from the Cartesian Hessian matrix through the Seminario method ${ }^{[29]}$ employing the MCPB.py module[30] of AmberTools19. [24] The dihedral potentials of cisplatin were neglected because metal-ligand torsion barriers are usually below the thermal energy. ${ }^{[31]}$ The atomic charges and Lennard-Jones parameters were taken from previous simulations. ${ }^{[19]}$ All the parameters for cisplatin are listed in Table 1. All the simulations described below have been evolved by the pmemd CUDA implementation ${ }^{[32]}$ of the Amber18 program. ${ }^{[24]}$

Table 1. Force field parameters for cisplatin: bond force constants $k_{b}$ equilibrium distances $R_{0}$, angle force constants $k_{a}$, equilibrium angles $\theta_{0}$, atomic charges $q$ and Lennard-Jones parameters $R_{\min } / 2$ and $\varepsilon$.

\begin{tabular}{|c|c|c|c|}
\hline \multicolumn{4}{|c|}{ Bond Parameters } \\
\hline Bond & $k_{b}\left(\mathrm{kcal} /\left[\mathrm{mol} \AA^{2}\right]\right)$ & & $R_{0}(\AA)$ \\
\hline $\mathrm{Pt}-\mathrm{Cl}$ & 123.100 & & 2.305 \\
\hline Pt-N & 88.900 & & 2.111 \\
\hline $\mathrm{N}-\mathrm{H}$ & 392.400 & & 1.019 \\
\hline \multicolumn{4}{|c|}{ Angle Parameters } \\
\hline Angle & $k_{a}\left(\mathrm{kcal} /\left[\mathrm{mol} \mathrm{rad}^{2}\right]\right)$ & & $\theta_{0}$ (degrees) \\
\hline $\mathrm{N}-\mathrm{Pt}-\mathrm{H}$ & 132.790 & & 98.410 \\
\hline Cl-Pt-N $\mathrm{N}_{\text {cis }}$ & 105.950 & & 83.010 \\
\hline $\mathrm{Cl}-\mathrm{Pt}-\mathrm{N}_{\text {trans }}$ & 106.350 & & 178.580 \\
\hline $\mathrm{Cl}-\mathrm{Pt}-\mathrm{Cl}$ & 88.200 & & 95.570 \\
\hline $\mathrm{Pt}-\mathrm{N}-\mathrm{H}$ & 52.880 & & 110.140 \\
\hline $\mathrm{H}-\mathrm{N}-\mathrm{H}$ & 41.400 & & 106.40 \\
\hline \multicolumn{4}{|c|}{ Intermolecular parameters } \\
\hline Atom & $q$ (a.u.) & $R_{\min } / 2(\AA)$ & $\varepsilon(\mathrm{kcal} / \mathrm{mol})$ \\
\hline $\mathrm{Pt}$ & 0.069 & 2.053 & 1.055 \\
\hline $\mathrm{Cl}$ & -0.375 & 2.597 & 0.038 \\
\hline $\mathrm{N}$ & -0.410 & 1.896 & 0.045 \\
\hline $\mathrm{H} 1$ & 0.239 & 0.052 & 0.018 \\
\hline $\mathrm{H} 2$ & 0.256 & 0.052 & 0.018 \\
\hline
\end{tabular}

Once the system was set up, the following step was to equilibrate the structure and the density of the solvated bilayer. At first, the whole system 
was minimized using the steepest descent method for 5000 steps, and the conjugate gradient method for another 5000 steps. Then, it was gradually heated in the canonical (NVT) ensemble, employing a Langevin thermostat with a collision frequency of $1 \mathrm{ps}^{-1}$, to $300 \mathrm{~K}$ for $300 \mathrm{ps}$. Positional restraints were imposed on the lipid bilayer and on the cisplatin molecule during the heating process, by applying force constants of 10 $\mathrm{kcal} /\left(\mathrm{mol}^{2}\right)$ and $5 \mathrm{kcal} /\left(\mathrm{mol}^{2}\right)$, respectively. Once the system was at 300 $\mathrm{K}$, three consecutive molecular dynamics simulations of 4 ns each were performed at constant pressure (isothermal-isobaric NPT ensemble), in which the positional restraints on the lipid were gradually decreased by applying force constants of $10 \mathrm{kcal} /\left(\mathrm{mol}^{2}\right), 5 \mathrm{kcal} /\left(\mathrm{mol} \AA^{2}\right)$ and 0 $\mathrm{kcal} /\left(\mathrm{mol} \AA^{2}\right)$, correspondingly, whereas the force constant of 5 $\mathrm{kcal} /\left(\mathrm{mol} \AA^{2}\right)$ applied on the cisplatin molecule was kept throughout the three NPT simulations. The Berendsen barostat with a pressure relaxation time of 1 ps was employed to maintain the pressure around 1 bar. Finally, a 100 ns production simulation was performed in the NPT ensemble where the restrain force constant of the cisplatin was maintained at $5 \mathrm{kcal} /\left(\mathrm{mol} \AA^{2}\right)$ For all the steps of this protocol the Particle Mesh Ewald method ${ }^{[33]}$ was used to calculate the Coulomb interactions, where the direct-space sum was limited to a cutoff of $10 \AA$. For the computation of van der Waals interactions the same cutoff was applied. In addition, bond distances involving $\mathrm{H}$ atoms were restrained by using the SHAKE algorithm ${ }^{[34]}$ and a time step of 2 fs was employed.

After the equilibration of the system, the permeation of cisplatin through the DOPC lipid bilayer was simulated by umbrella sampling molecular dynamics. The reaction coordinate was defined as the distance between the centre of mass of cisplatin and the centre of mass of the membrane along the $z$ axis (normal to the membrane), as schematically shown in Figure $1 \mathrm{~A}$. The initial value of the reaction coordinate was $32.0 \AA$. This distance was divided into 65 windows separated by $0.5 \AA$. A molecular dynamics simulation of 15 ns was carried out at constant pressure (NPT) on each of the windows applying a harmonic bias potential with a force constant of $2.5 \mathrm{kcal} /\left(\mathrm{mol}^{2}\right)$ on the reaction coordinate. Therefore, a total time of 975 ns was run to simulate the permeation of cisplatin. These simulations were performed sequentially, i.e., the initial conditions (geometry and velocities) of a window were selected from the last snapshot of the previous window. The same technical parameters employed for the $100 \mathrm{~ns}$ production described above were used in the simulation of each window. The free-energy profile was obtained by means of the WHAM approach. ${ }^{[35]}$ The energy-decomposition, interaction-energy and hydrogen bonding analyses were performed using the cpptra module ${ }^{[36]}$ of the AmberTools19 suite. ${ }^{[24]}$ Visual Molecular Dynamics ${ }^{[37]}$ was used to visualize the trajectories.

\section{Acknowledgements}

We thank the Comunidad de Madrid for funding through the Attraction of Talent Program (Grant Ref. 2018-T1/BMD-10261). The Centro de Computación Científica (CCC) of Universidad Autónoma de Madrid is thanked for generous computational resources.

\section{Keywords: Cisplatin • Lipid Bilayer $\cdot$ Permeation $•$ Umbrella} Sampling $\cdot$ Molecular Dynamics

[1] B. Rosenberg, L. Van Camp, T. Krigas, Nature 1965, 205, 698-699.

[2] B. Rosenberg, L. VanCamp, J. E. Trosko, V. H. Mansour, Nature 1969 222, 385-386.

[3] aE. R. Jamieson, S. J. Lippard, Chem. Rev. 1999, 99, 2467-2498; bL. Kelland, Nat. Rev. Cancer 2007, 7, 573-584.

[4] aS. E. Sherman, D. Gibson, A. H. J. Wang, S. J. Lippard, Science 1985, 230, 412-417; bP. M. Takahara, A. C. Rosenzweig, C. A. Frederick, S. J. Lippard, Nature 1995, 377, 649-652; cC. A. Rabik, M. E. Dolan, Cancer Treat. Rev. 2007, 33, 9-23; dF. Legendre, V. Bas, J. Kozelka, J. C.
Chottard, Chem. Eur. J. 2000, 6, 2002-2010; eN. Martinho, T. C. B Santos, H. F. Florindo, L. C. Silva, Front. Physiol. 2019, 10, 1898; fS Dasari, P. Bernard Tchounwou, Eur. J. Pharmacol. 2014, 740, 364-378; gT. Makovec, Radiol. Oncol. 2019, 53, 148-158.

[5] aY. Mantri, S. J. Lippard, M. H. Baik, J. Am. Chem. Soc. 2007, 129, 5023 5030; bM. H. Baik, R. A. Friesner, S. J. Lippard, J. Am. Chem. Soc. 2003 125, 14082-14092; cJ. Raber, C. Zhu, L. A. Eriksson, J. Phys. Chem. B 2005, 109, 11006-11015; dS. Sharma, P. Gong, B. Temple, D. Bhattacharyya, N. V. Dokholyan, S. G. Chaney, J. Mol. Biol. 2007, 373, 1123-1140; eT. Rivel, C. Ramseyer, S. Yesylevskyy, Sci. Rep. 2019, 9 5627; fS. Yesylevskyy, T. Rivel, C. Ramseyer, Sci. Rep. 2019, 9, 17214. [6] S. A. Aldossary, Biomed. Pharmacol. J. 2019, 12, 7-15.

[7] aS. Dilruba, G. V. Kalayda, Cancer Chemother. Pharmacol. 2016, 77, 1103-1124; bT. C. Johnstone, G. Y. Park, S. J. Lippard, Anticancer Res 2014, 34, 471-476; cJ. Czapla-Masztafiak, J. J. Nogueira, E. Lipiec, W. M. Kwiatek, B. R. Wood, G. B. Deacon, Y. Kayser, D. L. A. Fernandes, M. V. Pavliuk, J. Szlachetko, L. González, J. Sá, J. Phys. Chem. Lett. 2017, 8, 805-811; dQ. Chen, Y. Yang, X. Lin, W. Ma, G. Chen, W. Li, X. Wang, Z. Yu, Chem. Comm. 2018, 54, 5369-5372; eR. G. Kenny, S. W. Chuah, A. Crawford, C. J. Marmion, Eur. J. Inorg. Chem. 2017, 2017, 1596-1612; fS. Ramachandran, B. Temple, A. N. Alexandrova, S. G. Chaney, N. V. Dokholyan, Biochemistry 2012, 51, 7608-7617; gD. Veclani, A. Melchior, M. Tolazzi, J. P. Cerón-Carrasco, J. Am. Chem. Soc. 2018, 140, 14024-14027.

[8] S. Harrach, G. Ciarimboli, Front. Pharmacol. 2015, 6, 85

[9] N. D. Eljack, H. Y. M. Ma, J. Drucker, C. Shen, T. W. Hambley, E. J. New, T. Friedrich, R. J. Clarke, Metallomics 2014, 6, 2126-2133.

[10] J. Kozelka, Inorg. Chim. Acta 2009, 362, 651-668.

[11] Z. H. Siddik, Oncogene 2003, 22, 7265-7279.

[12] S. H. Chen, J. Y. Chang, Int. J. Mol. Sci. 2019, 20, 4136.

[13] Y. Zhang, W. Zeng, F. Jia, J. Ye, Y. Zhao, Q. Luo, Z. Zhu, F. Wang, Surf. Interface Anal. 2020, 52, 256-263.

[14] S. Ramachandran, A. P. Quist, S. Kumar, R. Lal, Langmuir 2006, 22, 8156-8162.

[15] A. Rebillard, X. Tekpli, O. Meurette, O. Sergent, G. LeMoigne-Muller, L. Vernhet, M. Gorria, M. Chevanne, M. Christmann, B. Kaina, L. Counillon, E. Gulbins, D. Lagadic-Gossmann, M. T. Dimanche-Boitrel, Cancer Res. 2007, 67, 7865-7874.

[16] T. C. Johnstone, K. Suntharalingam, S. J. Lippard, Chem. Rev. 2016 116, 3436-3486.

[17] G. P. Stathopoulos, T. Boulikas, J. Drug Deliv. 2012, 2012, 581363.

[18] G. van Meer, A. I. P. M. de Kroon, J. Cell Sci. 2011, 124, 5-8.

[19] S. Yesylevskyy, B. Cardey, S. Kraszewski, S. Foley, M. Enescu, A. M. da Silva, H. F. D. Santos, C. Ramseyer, J. Mol. Model. 2015, 21, 268.

[20] aJ. J. Nogueira, M. Meixner, M. Bittermann, L. González, ChemPhotoChem 2017, 1, 178-182; bP. A. Sánchez-Murcia, J. J. Nogueira, L. González, J. Phys. Chem. Lett. 2018, 9, 683-688; cC. T. Lee, J. Comer, C. Herndon, N. Leung, A. Pavlova, R. V. Swift, C. Tung C. N. Rowley, R. E. Amaro, C. Chipot, Y. Wang, J. C. Gumbart, J. Chem Inf. Model. 2016, 56, 721-733.

[21] J. Kästner, WIREs: Comput. Mol. Sci. 2011, 1, 932-942.

[22] aM. D. De Vetta, L. González, J. J. Nogueira, ChemistryOpen 2018, 7 475-483; bQ. Gong, H. Zhang, C. Chen, RSC Adv. 2019, 10, 790-800.

[23] S. Jo, T. Kim, V. G. Iyer, W. Im, J. Comput. Chem. 2008, 29, 1859-1865

[24] D. A. Case, I. Y. Ben-Shalom, S. R. Brozell, D. S. Cerutti, T. E. Cheatham III, V. W. D. Cruzeiro, T. A. Darden, R. E. Duke, D. Ghoreishi, M. K. Gilson, H. Gohlke, A. W. Goetz, D. Greene, R. Harris, N. Homeyer, Y. Huang, S. Izadi, A. Kovalenko, T. Kurtzman, T. S. Lee, S. LeGrand, P. Li, C. Lin, J. Liu, T. Luchko, R. Luo, D. J. Mermelstein, K. M. Merz, Y. Miao, G. Monard, C. Nguyen, H. Nguyen, I. Omelyan, A. Onufriev, F. Pan, R. Qi, D. R. Roe, A. Roitberg, C. Sagui, S. Schott-Verdugo, J. Shen, C. L. Simmerling, J. Smith, R. Salomon-Ferrer, J. Swails, R. C. Walker, J Wang, H. Wei, R. M. Wolf, X. Wu, L. Xiao, D. M. York, P. A. Kollman, University of California, San Francisco, 2018.

[25] Å. A. Skjevik, B. D. Madej, R. C. Walker, K. Teigen, J. Phys. Chem. B 2012, 116, 11124-11136.

[26] C. J. Dickson, B. D. Madej, Å. A. Skjevik, R. M. Betz, K. Teigen, I. R. Gould, R. C. Walker, J. Chem. Theory Comput. 2014, 10, 865-879. 
[27] W. L. Jorgensen, J. Chandrasekhar, J. D. Madura, R. W. Impey, M. L. Klein, J. Chem. Phys. 1983, 79, 926-935.

[28] P. Li, L. F. Song, K. M. Merz, J. Chem. Theory Comput. 2015, 11, 16451657.

[29] J. M. Seminario, Int. J. Quantum Chem. 1996, 60, 1271-1277.

[30] P. Li, K. M. Merz, J. Chem. Inf. Model. 2016, 56, 599-604.

[31] P. Li, K. M. Merz, Chem. Rev. 2017, 117, 1564-1686.

[32] R. Salomon-Ferrer, A. W. Götz, D. Poole, S. Le Grand, R. C. Walker, J. Chem. Theory Comput. 2013, 9, 3878-3888.

[33] M. F. Crowley, T. A. Darden, T. E. Cheatham lii, D. W. Deerfield li, J. Supercomput. 1997, 11, 255-278.

[34] S. Miyamoto, P. A. Kollman, J. Comput. Chem. 1992, 13, 952-962.

[35] aS. Kumar, J. M. Rosenberg, D. Bouzida, R. H. Swendsen, P. A. Kollman, J. Comput. Chem. 1992, 13, 1011-1021; bA. Grossfield.

[36] D. R. Roe, T. E. Cheatham, J. Chem. Theory Comput. 2013, 9, 3084 3095

[37] W. Humphrey, A. Dalke, K. Schulten, J. Mol. Graphics 1996, 14, 33-38. 